\title{
INTEGRATION OF KRAFT PULPING ON A FOREST BIOREFINERY BY THE ADDITION OF A STEAM EXPLOSION PRETREATMENT
}

\author{
Raquel Martin-Sampedro, Maria E. Eugenio, Esteban Revilla, Juan A. Martín, and \\ Juan C. Villar*
}

\begin{abstract}
Steam explosion has been proposed for a wide range of lignocellulosic applications, including fractionation of biomass, pre-treatment of biomass for ethanol production, or as an alternative to conventional mechanical pulping. Nevertheless, a steam explosion process could also be used as pretreatment before chemical pulping, expecting a reduction in cooking time due to the open structure of the exploded chips. Thus, to evaluate the effect of steam explosion as a pretreatment in the kraft pulping of Eucalyptus globulus, steam exploded chips and control chips were subjected to kraft cookings. Steam exploded chips provided pulps with reductions of kappa number by up to $70 \%$ with no significant change in viscosity. Therefore, the cooking time could be shortened by $60 \%$, increasing the productivity and obtaining pulps with similar delignification degree to those of the control pulp. Furthermore, not only the production rate could be increased, but also most of the hemicelluloses could be recovered before pulping and converted to a value-added product. Finally, although exploded pulp had inferior mechanical strength, the optical properties, which are more important in eucalyptus pulps, were found to be better.
\end{abstract}

Keywords: Eucalyptus globulus; Forest biorefinery; Kraft pulping; Steam explosion; Pulp properties

Contact information: Instituto Nacional de Investigación y Tecnología Agraria y Alimentaria INIA. Carretera de la Coruña, km 7.5, 28040, Madrid (Spain); *Corresponding author: villar@inia.es

\section{INTRODUCTION}

The concept of a forest biorefinery is based on the use of lignocellulosic biomass as raw material in the integrated production of fuels, energy, and chemical products. The possibility of transforming a chemical pulp mill into a forest biorefinery is attracting widespread research interest. Such a transformed mill could produce higher value-added products such as ethanol, polymers, carbon fibers, and diesel fuel, in addition to pulp (San Martin et al. 1995; Van Heiningen 2006; Ragauskas et al. 2006). One of the ways to achieve this goal is to extract a portion of the hemicelluloses from wood prior to pulping, obtaining a liquid fraction that could be converted into ethanol and/or other chemical products. The hemicelluloses extraction could be done by different treatments, such as acid hydrolysis, autohydrolysis, steam explosion, or alkali extraction; however, steam explosion treatment shows some advantages that make it a competitive process to extract hemicelluloses. 
The steam explosion process consists of treatment of lignocellulosic material with high-pressure steam, followed by a rapid decompression, which forces the fibrous material to "explode" into separated fibers and fiber bundles. When no other chemicals are added to the process, the high-temperature steam (due to the high rate of dissociation of water) leads to the release of acids from the acetylated wood components; the released acids then catalyze hydrolytic reactions in the wood polymers. These autohydrolysis reactions result in a loss of hemicellulose and a decrease in the content of $\beta-\mathrm{O}-4$ structures in lignin (Robert et al. 1988; Jakobsons et al. 1995; Josefsson et al. 2001, 2002). These chemical changes depend greatly on the temperature used and the treatment time (Chua and Wayman 1979).

The earliest application of this process was developed by Mason in 1928. This author proposed using a steam treatment followed by an explosive decompression for the production of Masonite board stock (Mason 1928). Another application was suggested by Babcock (1932), who used steam explosion as a pretreatment method for the production of ethanol. Steam explosion has also been proposed as an alternative to improve conventional mechanical or chemo-mechanical pulping methods (Delong 1987). During this process, lignin is softened to allow higher defibration without excessive damage and/or degradation to individual fibers. However, the process has not been industrially implemented (Heitner et al. 1993) because pulps so produced have not shown any superior properties over those from chemimechanical pulp (CMP) or chemithermomechanical pulp (CTMP).

As has been mentioned before, steam explosion could be also used to remove hemicelluloses, since during the steam treatment, hemicelluloses are depolymerized, partially degraded, and solubilized in hot water, yielding a mixture of monomeric and oligomeric sugars (Puls et al. 1985). Removed hemicelluloses can be a valuable source of hexose and pentose sugars, and they can be further converted into value-added products such as ethanol, polymers, and other chemicals (Li et al. 2010).

Removal of hemicelluloses from wood as a pretreatment step is being practiced commercially in the production of dissolving pulps, where the hemicelluloses are removed to allow the production of pure cellulose (Wafa Al-Dajani and Tschirner 2008). The most common commercial procedure for extracting hemicelluloses is pre-steaming to release natural wood acids (autohydrolysis) followed by water extraction or acid hydrolysis with small amounts of mineral acids (sulfuric acid or hydrochloric acid). Compared to these processes, steam explosion not only allows the recovery of wood components without their degradation (Avellar and Glasser 1998; Britt 1970), but also leaves a solid residue (exploded chips) that is expected to be pulped more easily than those obtained conventionally due to the open structure of the exploded chips that might encourage an efficient diffusion of cooking liquor into the fibers (Ahvazi et al. 2007). Therefore, taking into account the advantages of the steam explosion pretreatment, it can be concluded that this process could be used as an efficient pretreatment in pulping, increasing the total revenue stream for the pulp industry and converting the pulp mill into an integrated forest biorefinery (Ragauskas et al. 2006; Van Heiningen 2006).

Although some studies have assessed the pre-extraction of hemicelluloses before kraft pulping (Li et al. 2010; Wafa Al-Dajani et al. 2009; San Martin et al. 1995; Ahvazi et al. 2007), few of them have evaluated the potential of steam explosion to this end. 
Therefore, deeper research would be needed to evaluate not only the effect of steam explosion pretreatment on kraft pulping, but also the potential recovery of sugars from the liquid fraction generated during this pretreatment.

The aim of this work was to evaluate the possibility of integrating a kraft pulping into a forest biorefinery, studying the sugar recovery and the effect of steam explosion on kraft pulping and pulp properties. We selected Eucalyptus globulus because it is the raw material most commonly used to produce kraft pulp in Spain, and also because it has a high content of hemicelluloses (pentosan content $17.9 \%$ on o.d. wood).

\section{EXPERIMENTAL}

\section{Steam Explosion Treatment}

Eucalyptus globulus chips were kindly provided by La Montañanesa pulp mill (Torraspapel group, Spain). After two days conditioning in the laboratory (inside polyethylene bags) to guaranty the chips homogenization, chips were divided in two groups. One of them was immersed in water at $25^{\circ} \mathrm{C}$ (pre-extraction) during 16 hours to evaluate the effect of the uniformity of impregnation on the subsequent steam explosion treatment.

The steam explosion treatment was performed in a 26 liter stainless steel digester (manufactured by Cadepla S. L.) capable of achieving $190{ }^{\circ} \mathrm{C}$ temperature and $16 \mathrm{Kg} / \mathrm{cm}^{2}$ pressure. The digester is connected to a blowing tank into which chips are discharged at the end of the treatment. In each experiment, $500 \mathrm{~g}$ of E. globulus chips were treated with steam at $183^{\circ} \mathrm{C}\left(10 \mathrm{Kg} / \mathrm{cm}^{2}\right)$ during 5 or 10 minutes and discharged at $6 \mathrm{Kg} / \mathrm{cm}^{2}$. The number of cycles was studied to prove that the improvement of the accessibility of the exploded chips (due to their open structure) is more influenced by the final explosion than by the steam treatment. When a second cycle was carried out, the exploded chips were treated with steam again during 3 minutes and discharged into the blowing tank. Table 1 summarizes the operational conditions applied.

The severity factor of each treatment was calculated according to the following equation defined by Overend and Chornet (1987).

$$
\mathrm{S}_{0}=\log \left(\mathrm{e}^{\frac{\mathrm{T}-100}{14.75}} \cdot \mathrm{t}\right)
$$

in which $\mathrm{T}$ is the temperature $\left({ }^{\circ} \mathrm{C}\right)$ and $\mathrm{t}$ the duration of the treatment (min).

The treated chips were then removed from the blowing tank, washed with cold water, air-dried, and finally weighted. The water content was measured to determine the total yield of the steam explosion process.

The water retention, or hydration capacity, of the chips was the weight of water absorbed by the chips after being immersed for 6 hours, and it was expressed as grams of water per 100 grams of dry wood.

The pretreatment that provided the highest water retention was selected for further studies. The pretreated chips were analyzed to determine: acetone extractives (UNE-EN ISO 14453), hot water extractives (UNE 57-013), lignin content (TAPPI T 222 om-88; UNE 57-100), and holocellulose content (Wise et al. 1946). Chips from control A were 
also analyzed as reference. Also, the liquid fraction of the selected steam explosion was recovered to determine the sugar content. An aliquot $(1 \mathrm{~mL})$ was filtered through 0.45 $\mu \mathrm{m}$ membranes and used for direct HPLC determination of monosaccharides and acetic acid. A second aliquot of $25 \mathrm{~mL}$ was subjected to quantitative posthydrolysis with $4 \%$ $\mathrm{H}_{2} \mathrm{SO}_{4}$, at $120^{\circ} \mathrm{C}$, for $60 \mathrm{~min}$., before HPLC analysis. The increase in the concentrations of monosaccharides and acetic acid caused by posthydrolysis was used as a measure of the concentrations of oligomers and acetyl groups bound to oligosaccharides, respectively (Garrote et al. 1999).

Table 1. Steam Explosion Conditions

\begin{tabular}{ccccc}
\hline Sample & Pre-extraction & $\begin{array}{c}\text { Number of } \\
\text { cycles }\end{array}$ & $\begin{array}{c}\text { Time of first } \\
\text { cycle }(\mathrm{min})\end{array}$ & $\begin{array}{c}\text { Severity factor } \\
\left(\mathrm{S}_{0}\right)\end{array}$ \\
\hline Control A & No & - & - & - \\
Control B & Yes & - & - & - \\
SE1 & No & 1 & 5 & 3.14 \\
SE2 & Yes & 1 & 5 & 3.14 \\
SE3 & No & 1 & 10 & 3.44 \\
SE4 & Yes & 1 & 10 & 3.44 \\
SE5 & No & 2 & 5 & 3.35 \\
SE6 & Yes & 2 & 5 & 3.35 \\
SE7 & No & 2 & 10 & 3.56 \\
SE8 & Yes & 2 & 10 & 3.56 \\
\hline
\end{tabular}

\section{Kraft Cooking Process}

Kraft cooking was performed on the chips pretreated by steam explosion and on those of control A in four 1-liter pressurized reactors. Reactors were placed in a $20 \mathrm{~L}$ rotatory pressurized vessel that contained hot water for indirect heating of the reactors. The rotatory vessel had a jacket-type electrical heater controlled by a computer to set the cooking temperature. Cooking conditions (referred to steam exploded chips, or non exploded chips in the control case) were: $60 \mathrm{~g}$ of dry chips, $4 \mathrm{~L} / \mathrm{Kg}$ liquor to wood ratio, $16 \%$ active alkali, $20 \%$ sulfidity, $160{ }^{\circ} \mathrm{C}$ cooking temperature, and 60 minutes to maximum temperature. Time at maximum temperature was varied from 24 to 56 minutes to obtain an $\mathrm{H}$-factor ranging from 180 to 400 (a control parameter in the pulping process which includes time and temperature as a single variable). The $\mathrm{H}$-factor was calculated according to the following equation:

$$
\mathrm{H}=\int_{0}^{\mathrm{t}} \mathrm{e}^{\left(43.2-\frac{16115}{\mathrm{~T}}\right)} \mathrm{dt}
$$

in which $\mathrm{T}$ is the temperature $(\mathrm{K})$ and $\mathrm{t}$ the time (hours).

After each batch, the reactors were removed from the vessel, and the cooked chips were washed, disintegrated and screened to determine their reject content and screened and total kraft yields based on the solids content of the material submitted to kraft 
pulping, whether steam-exploded or not. Moreover, the overall yield has been calculated taking into account both the steam explosion and the kraft processes. Black liquor was titrated with $\mathrm{HCl}$ to determine the chemical reagent consumption. The kappa number (TAPPI T-236/UNE 57034), viscosity (UNE 57039-1), and hexenuronic acid content (Gellersted and Li. 1996) were determined in the pulp samples. Also, the pulp quality was assessed in an optical microscope (Vanox AH3 Olympus connected to a Color View III digital camera).

The cooking process that produced the best pulp of those that were assayed was scaled up, changing the liquid-wood ratio and the active alkali because of an equipment limitation, as it will be explained below. Kraft cooking was done in a $26 \mathrm{~L}$ batch reactor furnished with a system for recirculation and heating of the cooking liquor. The cooking temperature was controlled by a computer running specially developed software. Due to the volume of the liquor recirculation circuit and to the characteristics of the steam exploded chips, the liquid-wood ratio had to be increased to $6 \mathrm{~L} / \mathrm{Kg}$ in order to assure a complete immersion of the chips. The active alkali was also raised to $18 \%$ to approach the concentration in the small reactors, since a greater volume of water was used. Other cooking conditions were fixed as follows: $1000 \mathrm{~g}$ of dry chips, $20 \%$ sulfidity, $160{ }^{\circ} \mathrm{C}$ cooking temperature, 40 minutes to maximum temperature, and 27 minutes at cooking temperature (H-Factor of 200).

After the cooking process was completed, the chips were discharged into the blowing tank, washed, disintegrated, and screened to determine their reject content, screened and total yields, kappa number (ISO 302), and pulp viscosity (ISO 5351-1).

Control pulp, obtained from chips that were not subjected to the steam explosion pretreatment, had a high lignin content, needing further delignification to be comparable with the pulp from pretreated chips. Therefore, oxygen delignification was applied to this pulp in a $20 \mathrm{~L}$ rotatory reactor. Conditions of this treatment were: $0.6 \mathrm{MPa}$ oxygen pressure, $10 \%$ consistency, $5 \% \mathrm{NaOH}$ (on dry pulp), $0.5 \% \mathrm{MgSO}_{4}$ (on dry pulp), and 60 minutes of treatment at $110{ }^{\circ} \mathrm{C}$. Control pulp characteristics were evaluated as previously described for the other pulps.

\section{Refining and Handsheets Characterization}

The degree of refining (Schopper-Riegler) (UNE-EN ISO 5267-1) was determined in exploded and control pulps (after oxygen delignification). Pulps were subsequently refined in a PFI mill (UNE-EN ISO 5264-2/TAPPI T- 248) to different refining degrees $(1000,2000$, and 3000 revolutions) to obtain more conformable fibers for papermaking.

Handsheets were obtained from unrefined and refined pulps in accordance with ISO UNE-EN ISO 5269-2 and characterized in terms of apparent bulk density, tensile resistance, burst resistance, tear resistance (UNE-EN ISO 5270), brightness (UNE 57062), and yellowness index (TAPPI T-1216).

\section{Accelerated Ageing}

Exploded, control, and commercial pulps were subjected to accelerated ageing in order to study the evolution of their optical and mechanical properties and also the effect of the steam explosion pretreatment on this evolution. The accelerated ageing consisted 
of a moist heat treatment at $80^{\circ} \mathrm{C}$ and $65 \%$ relative humidity during 6 days, according to the standard UNE 57092-4.

\section{RESULTS AND DISCUSSION}

\section{Steam Explosion Treatment}

Different steam explosion pre-treatments were performed at $183^{\circ} \mathrm{C}$ by changing three variables, as shown in Table 1. Depending on the conditions, the yield varied between 94.3 and $79.3 \%$. The yield decreased with the intensity of the process, mainly depending on the treatment time (Fig. 1a). These results are consistent with those found by other authors (Wafa Al-Dajani et al. 2009; Li et al. 2010), who reported that the amount of hemicelluloses dissolved in the liquid fraction was higher when the autohydrolysis time increased.

The water retention capacity of the exploded chips could provide information about changes in the internal structure of the material and correlates with the intensity of the pretreatment. As shown in Fig. 1b, there was a significant increase in the water retention capacity of all materials after steam explosion. This fact suggests that the accessibility of the exploded chips was increased, which would also improve the diffusion of cooking liquor during a subsequent chemical pulping process. The effect was more pronounced for stronger treatments, for which the addition of a second steam explosion cycle was the most influential factor (maximum increase $90 \%$, comparing SE4 and SE8), followed by the treatment time (36\%, comparing SE2 and SE4). The cold water pre-extraction of the original material also increased the effect of steam explosion. This finding may have two causes: (i) the removal of extractives during this preextraction, which could make steam penetration easier and would improve chips' hydrophilicity; and (ii) the higher amount of water in the pre-extracted chips, which would produce a more intense explosion and chip opening.

a)

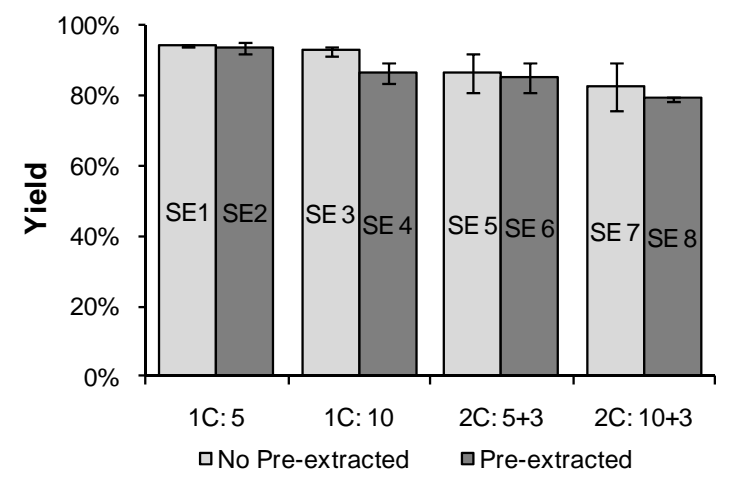

b)

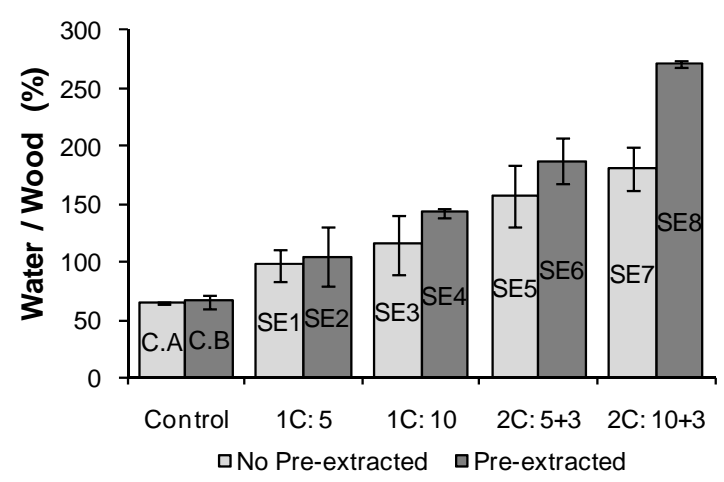

Fig. 1. Yield of steam explosion treatment (a) and water retention capacity of exploded chips (b)

Taking into account these results, the SE8 treatment, consisting of water preextraction followed by two steam explosion cycles of 10 and 3 minutes, was selected as the most appropriate pretreatment for kraft pulping. It provided chips with a more open 
structure that could be pulped more easily. The low yield obtained under these conditions would reduce the overall yield after the kraft process, but it would remove unaltered hemicelluloses that could be converted into value-added products, polymers, or ethanol for fuel (Li et al. 2010), increasing the total revenue stream for the pulp mill (Ragauskas et al. 2006; Van Heiningen 2006).

The chemical composition of the SE8 and the Control A samples, as well as the sugar content of the liquid fraction from the SE8 treatment are shown in Tables 2 and 3, respectively. It can be observed that there was an increase in the extractives content (water soluble and acetone soluble) and a reduction in the holocellulose content of the wood chips after steam explosion. The increase in extractives could be due to the fact that, during the steam explosion treatment, some macromolecules, such as lignin and polysaccharides, are partially degraded, becoming extractible in a subsequent solvent extraction. The holocellulose reduction was due to the hemicellulose degradation during the steam explosion treatment, as has been mentioned above. The sugar content in the liquid fraction obtained during this treatment also confirmed this hemicellulose degradation, since xylose was the main sugar present in this fraction. Eight percent of the original solid material (E. globulus) was recovered as xylose, after the SE8 treatment, and this could be used to produce value-added products.

Table 2. Chemical Composition of Control (Control A) and Exploded Chips (SE8)

\begin{tabular}{ccc}
\hline & Control A & SE8 \\
\hline Acetone extractives & $1.1 \%^{\mathrm{a}}$ & $4.9 \%^{\mathrm{a}}$ \\
Hot water extractives & $2.8 \%^{\mathrm{a}}$ & $7.5 \%^{\mathrm{a}}$ \\
Lignin & $18.0 \% \mathrm{\%}^{\mathrm{a}}$ & $18.4 \% \mathrm{\sigma}^{\mathrm{a}}$ \\
Holocellulose & $73.9 \%^{\mathrm{a}}$ & $64.7 \%^{\mathrm{a}}$ \\
Pentosans & $17.9 \%^{\mathrm{a}}$ & $10.4 \%^{\mathrm{a}}$ \\
Holocellulose / lignin ratio & 4.1 & 3.5 \\
\hline
\end{tabular}

${ }^{a}$ Referred to weight/weight over dry wood

Table 3. Sugar Contents of the Liquid Fraction from the Steam Explosion Treatment SE8

\begin{tabular}{ccc}
\hline & $\mathrm{g} / \mathrm{L}^{\mathrm{a}}$ & $\%$ extracted \\
\hline Acetyl groups & 1.7 & $4.6 \%$ \\
Glucose & 0.2 & $0.5 \%$ \\
Xylose & 3.0 & $8.0 \%$ \\
Arabinose & 0.04 & $0.1 \%$ \\
Total & 4.96 & $13.2 \%$ \\
\hline${ }^{\mathrm{a}}$ Including monomers and polymers \\
${ }^{\mathrm{b}}$ Weight of extracted sugar respect to weight of original material
\end{tabular}

\section{Kraft Pulping Process}

Non-pretreated chips (control A) and exploded chips (SE8) were pulped under the same conditions to compare the resulting pulps. The cooking time varied from 24 to 56 minutes (corresponding to an H-Factor varying from 180 to 400), in order to evaluate the effect of steam explosion at different cooking conditions. 
Total and screened kraft yields (without taking into account steam explosion yield; in other words, the yield calculation was based on the solids content of the material submitted to kraft pulping, whether steam-exploded or not) have been plotted versus the H-Factor in Fig. 2a and 2b, respectively. The total yield of both control and exploded samples decreased with the cooking time (higher H-Factor) because more lignin, and also more carbohydrates, were dissolved in the cooking liquor. Total and screened yields obtained with control chips were higher than those obtained with exploded chips in all cases, except from screened yields when the H-Factor was lower than 200. In order to explain this phenomenon, it has to be taken into account that when short pulpings were performed, control chips were not cooked long enough and a high amount of rejects was obtained; therefore a higher total yield and a lower screened yield were achieved. On the other hand, when longer pulping was carried out, control chips were cooked long enough, but exploded chips were overcooked, dissolving a high amount of carbohydrates in the cooking liquor and obtaining lower yields. However, the last comparison was made for the same H-factor, and the exploded and control chips did not need the same pulping intensity. Therefore, a comparison focusing on the kappa number of the pulps would be more appropriate.

In Fig. $2 \mathrm{c}$ and $2 \mathrm{~d}$ it can be observed that the steam explosion pretreatment increased the consumption of both $\mathrm{Na}_{2} \mathrm{~S}$ and $\mathrm{NaOH}$ during kraft pulping, in contrast to what has been reported by San Martin et al. (1995). However, this higher chemical consumption produced pulp with a lower kappa number (as it will be explained below), suggesting a higher efficiency of the chemical in delignification.

a)

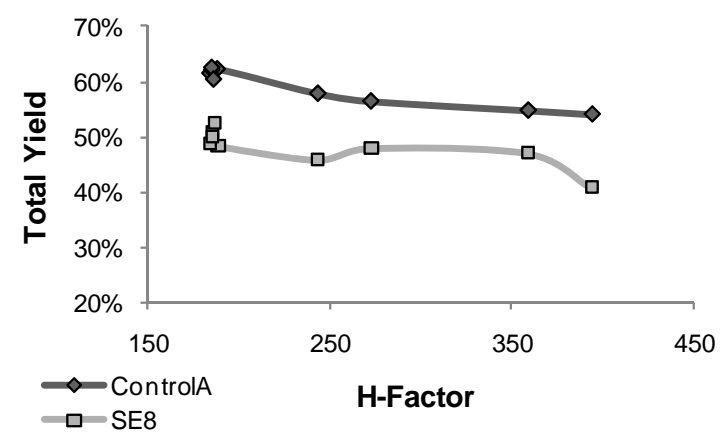

c)

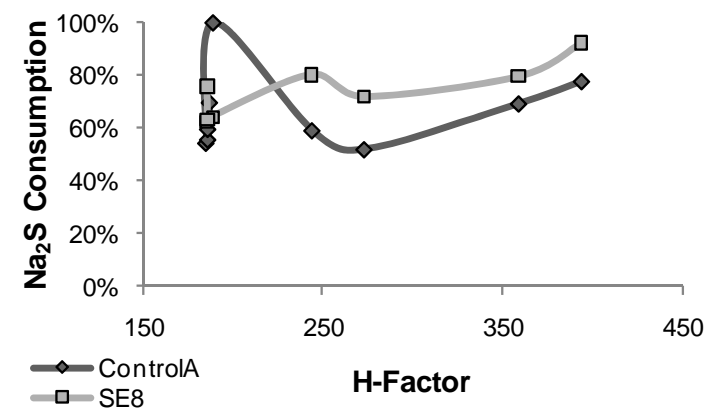

b)



d)

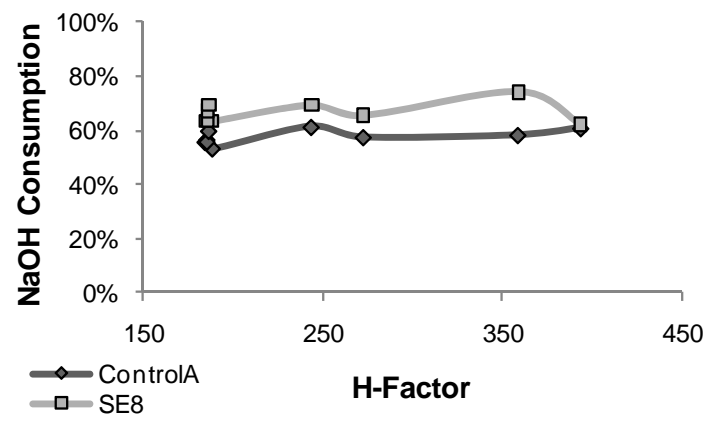

Fig. 2. Yields and chemical consumption during kraft pulping of control (Control $A-$ ) and exploded chips (SE8- - -): total yield (a), screened yield (b), $\mathrm{Na}_{2} \mathrm{~S}$ consumption (c), and $\mathrm{NaOH}$ consumption (d) 
In Fig. 3a and 3b, the kappa number and viscosity of exploded and control pulps are plotted versus the H-factor. Kappa numbers of exploded pulps were much lower than those obtained for control pulps due to the open structure of exploded chips, which increases the reagent accessibility. A maximum reduction of $70 \%$ in the kappa number (from 49 to 16) was observed when exploded chips were cooked under the mildest conditions (H-factor of 180). These results are similar to those found by San Martin et al. (1995), although they obtained a lower decrease in the kappa number (44\% from control pulp). Moreover, the kappa number decreased, as it was expected, when cooking was more intensive (higher $\mathrm{H}$-factor) because more lignin was removed. This drop in the $\mathrm{H}-$ factor was lower in the cases of exploded pulps because the kappa number obtained with an $\mathrm{H}$-factor of 180 was already low, and removing the residual lignin was more difficult and caused the degradation of more carbohydrates (providing a lower screened yield with longer pulping processes).

With regard to pulp viscosity, similar values were found in most of the samples and were comparable with the values reported for commercial pulps. However, the viscosity of the exploded pulp decreased dramatically when the H-Factor was higher than 360 due to overcooking of the chips, causing cellulose degradation (not observed in control chips). For an H-Factor of 270, the same viscosity was found for exploded and control pulps although the kappa number was much lower for the former (11.2 versus 30.4). Therefore, higher delignification could be obtained with exploded pulp without degrading carbohydrates.

Moreover, the content of hexenuronic acids (HexA) was found to be lower in exploded pulp than in control pulp (Fig. 3c). Taking into account that hemicelluloses are dissolved during the steam explosion pretreatment and that HexA are formed from xylans during kraft pulping, exploded chips gave pulps with lower HexA content. This could be a great advantage of the steam explosion pretreatment because the presence of HexA is detrimental to bleaching, since it increases the consumption of bleaching chemicals (Vuorinen et al. 1999; Ragnar 2001). On the other hand, the loss of hemicellulose in the steam explosion pretreatment could be detrimental to the refining and mechanical properties of the handsheets. This effect will be considered later in this article.

a)

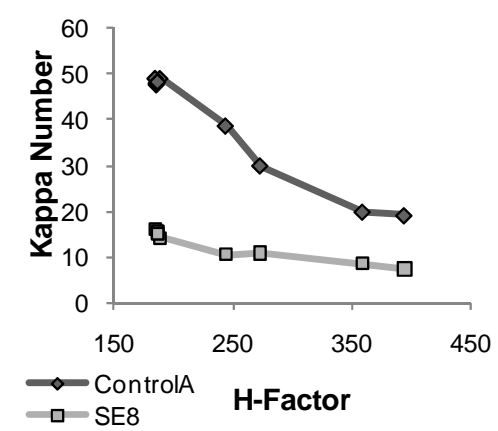

b)

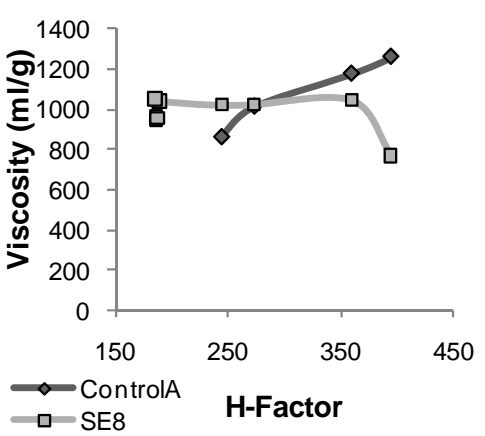

c)

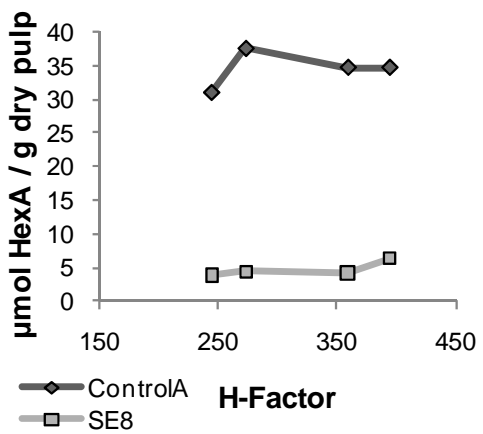

Fig. 3. Kappa number (a), viscosity (b) and hexenuronic acids content (c) of kraft pulps obtained from control (Control $A \smile$ ) and exploded chips (SE8- - ) 
In Fig. 4, fibers from exploded and control pulps can be observed. These pictures show that, after the 24 minute pulping process (H-factor of 190), control chips had not cooked long enough, presenting a high number of fiber bundles (shives). On the contrary, neither shives nor broken or degraded fibers were found in pulp produced from exploded chips.

a)

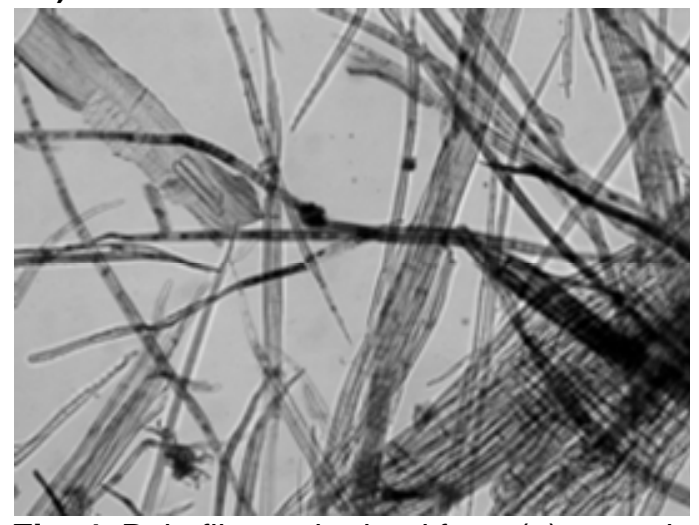

b)

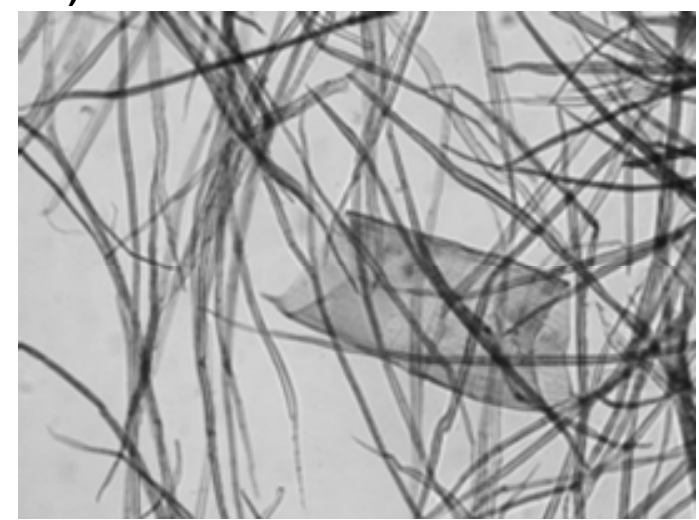

Fig. 4. Pulp fibers obtained from (a) control chips (Control A) and (b) steam exploded chips (SE8)

From all these results, an $\mathrm{H}$-factor of 200 (27 minutes at maximum temperature) was selected, because a more intensive cooking would overcook the steam exploded chips, as has been indicated above. This process was scaled up in a 26-L digester in order to evaluate the effect of the scale change as a first step prior to the industrial scale-up. The kappa number, the viscosity, and the total and screened kraft yield obtained for steam exploded (SE8) and untreated chips (control A) are shown in Table 4.

Tabla 4. Yields and Pulp Characteristics Obtained during Scaled Up Kraft Cooking and Subsequent Oxygen Delignification

\begin{tabular}{|c|c|c|c|c|}
\hline & SE8 & Control A & Control $\mathrm{A}+\mathrm{O}_{2}$ & Industrial \\
\hline Kappa Number & 15.5 & 50.6 & 26.4 & 14.0 \\
\hline Viscosity $(\mathrm{ml} / \mathrm{g})$ & 1166 & - & 915 & 1136 \\
\hline Screened Yield (\%) & 49.6 & 28.6 & $25.7^{\mathrm{b}}$ & - \\
\hline Total Yield $(\%)^{a}$ & 51.6 & 64.9 & - & - \\
\hline
\end{tabular}

A bigger difference in kraft yields between a steam-exploded and a control sample was obtained when pulping was scaled up. However, the difference in kappa number was found to be similar to that observed when pulping was performed in small reactors.

As was mentioned above, pulp obtained from untreated chips (Control A) had much higher lignin content than that obtained from exploded chips. In order to obtain a more comparable pulp in terms of refining and paper properties, the control pulp was subjected to oxygen delignification. In Table 4 it can be observed that, after this treatment, the kappa number decreased significantly, but the viscosity also decreased. However, with a steam explosion pretreatment, both a low kappa number and high 
viscosity could be simultaneously obtained. These results corroborate that steam explosion followed by a short kraft pulping pretreatment is a more efficient process than short kraft pulping followed by oxygen delignification.

From all these results, it can be concluded that, under the same cooking conditions, the steam exploded chips cooked faster and more efficiently than the untreated chips, or alternatively, they could be pulped at a lower temperature and/or with lower active alkali, while maintaining the same production rate (Ahvazi et al. 2007; San Martin 1995; Wafa Al-Dajani et al. 2009). With the first option, the cooking time could be reduced, increasing the production rate of the pulp mill. Moreover, as the hemicelluloses have been removed before pulping, the charge of solids in the recovery furnace has been reduced also and an increase of pulp production could be possible.

In these trials, the cooking time was reduced from 50 to 20 minutes to obtain a pulp of similar characteristics (kappa number $\sim 16$ and viscosity $\sim 1000 \mathrm{ml} / \mathrm{g}$ ) with a good yield $(+50 \%)$. Even though the overall pulp yield of the process (including steam explosion and kraft pulping) was lower $(\sim 40 \%)$, the pulp production of a hypothetic mill could be maintained at a similar level because of the increase in pulp production. Moreover, most of the hemicelluloses were removed before pulping without excessive degradation and converted into value-added products. Finally, since the exploded pulp had a very low HexA content, the consumption of chemicals in a subsequent bleaching process is expected to be reduced also.

\section{Refining and Handsheet Properties}

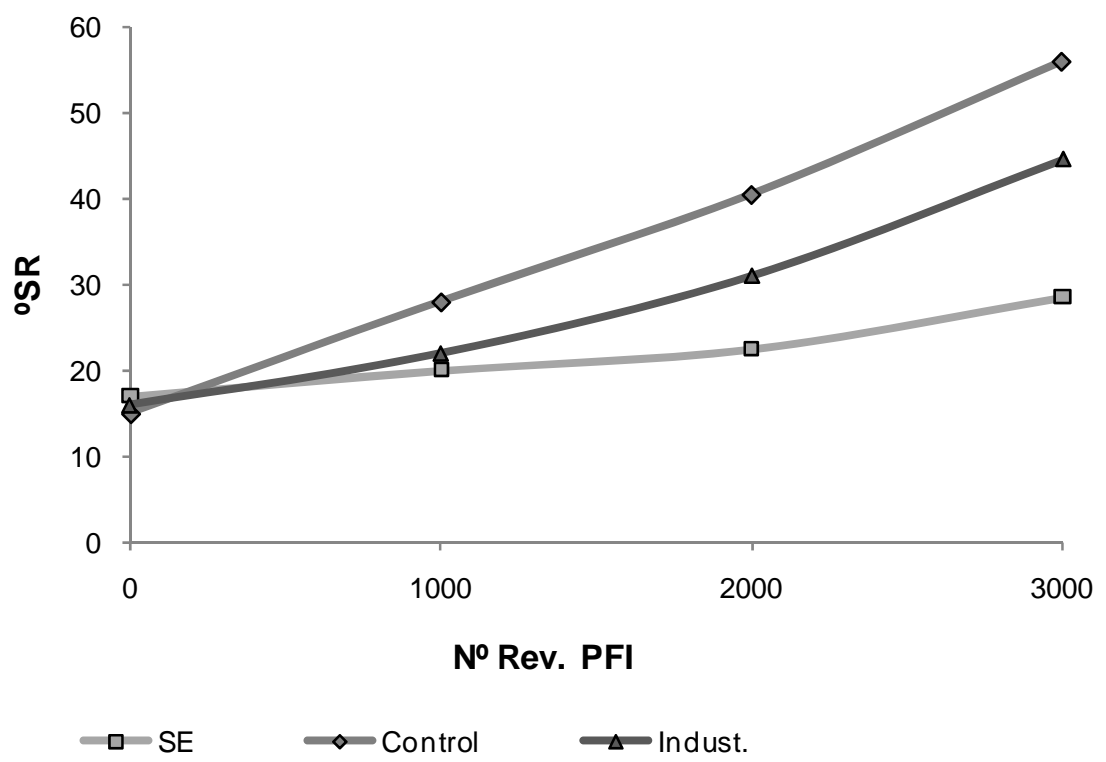

Fig. 5. Refining degree ('Schopper-Riegler) vs. refining intensity (PFI revolutions): SE $\square$-; Control $\checkmark$; Indust. $-\leftarrow$ 
The response of exploded pulp to refining was evaluated and compared with the control pulp response. As has been mentioned, before refining, the control pulp was subjected to oxygen delignification to obtain a pulp with lignin content similar to that of the steam explosion pulp. Moreover, an industrial pulp (kindly provided by Torraspapel group) was also refined and used as reference. The variation of the refining degree (measured as Schopper-Riegler) with the intensity of the PFI refining is plotted in Fig. 5.

It can be observed that the exploded pulp exhibited a slower increase of ${ }^{\circ} \mathrm{SR}$ with PFI revolutions than control and industrial pulps did. This means that the exploded pulp requires more energy to obtain a certain ${ }^{\circ} \mathrm{SR}$. This could be due to the removal of hemicelluloses during the steam explosion pretreatment, as has been mentioned above. Moreover, the control pulp showed a higher ${ }^{\circ} \mathrm{SR}$ for a given PFI revolution number compared to the industrial pulp studied.

Handsheets were formed from unrefined and refined control, industrial, and exploded pulps, and their mechanical and optical properties were determined. Results are shown in Fig. 6.

The unrefined steam exploded pulp showed a ${ }^{\circ} \mathrm{SR}$ of 17, similar to that obtained for unrefined control and industrial pulps (15 and $16{ }^{\circ} \mathrm{SR}$ respectively). Also, the mechanical properties were found to be similar to those of unrefined industrial pulp, apart from the tensile index. This index is related to the linking capacity between fibers, so the low value obtained for the exploded pulp could be due to the loss of hemicelluloses during the steam explosion pretreatment. Tear index was found to be similar for unrefined exploded and industrial pulps, which would indicate a similar degree of fiber degradation, consistent with what San Martin et al. (1995) have reported.

It can be observed also in Fig. 6 that the apparent bulk density increased with refining in a similar way for exploded, control, and industrial pulps.

The mechanical properties also increased with refining in all pulp samples, as was expected. Tensile and burst indexes were found similar for control and industrial pulps but lower for the exploded pulp. As has been mentioned above, this could be due to the removal of hemicelluloses during steam explosion.

Although control and industrial pulp tear indexes were higher than the exploded pulp tear index, the differences between them were lower than those observed in tensile and burst indexes. Unrefined exploded and industrial pulps gave similar values of tear index; however when refining was performed, the industrial pulp showed a better evolution, providing higher values of tear index.

In order to compare optical properties, the brightness, yellowness index, and opacity of dried pulp sheets were measured. The steam exploded pulp presented the best optical properties, since its yellowness index was found to be much lower than that of the control and industrial pulps, its opacity was higher than that of the control pulp, and its brightness was higher than that of the industrial pulp. Considering the evolution of the optical properties with refining, it can be observed that yellowness index and opacity did not change, but brightness decreased when the ${ }^{o} \mathrm{SR}$ increased. This could be caused by the homogenization of the pulp resulting from the split of the microscopic fiber bundles present in the pulps. 
a)

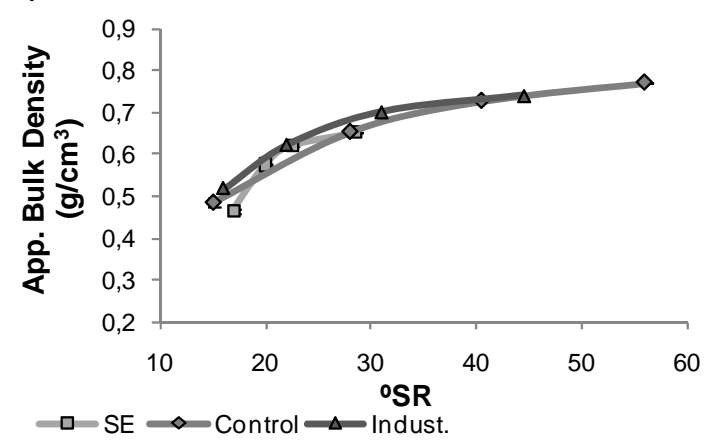

c)

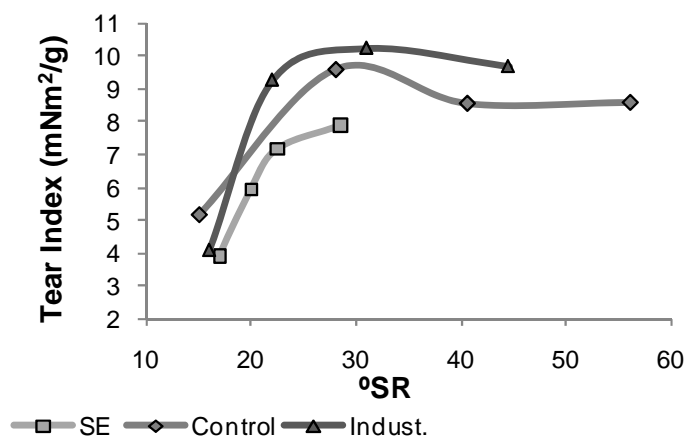

e)

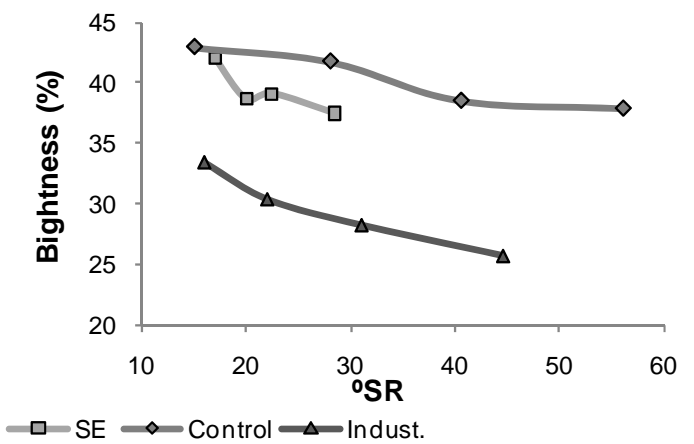

b)

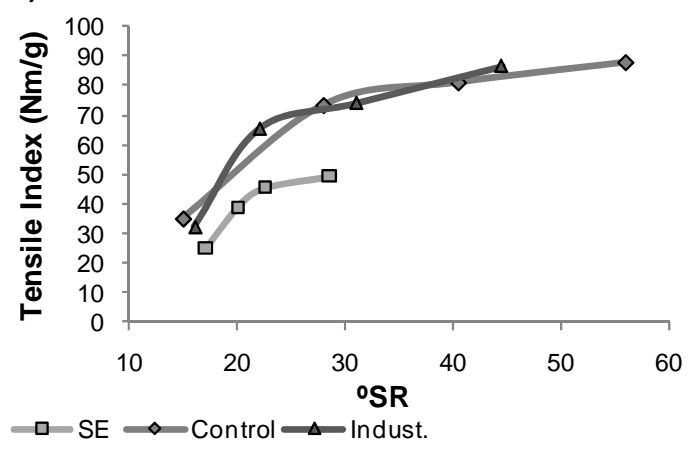

d)

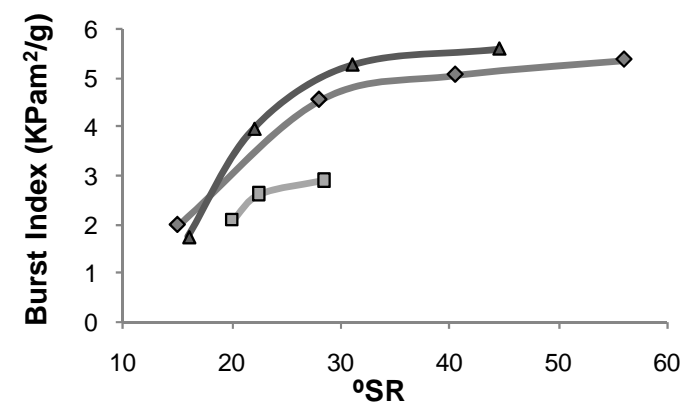

$\square-\mathrm{SE} \leadsto$ Control $\triangle$ Indust.

f)

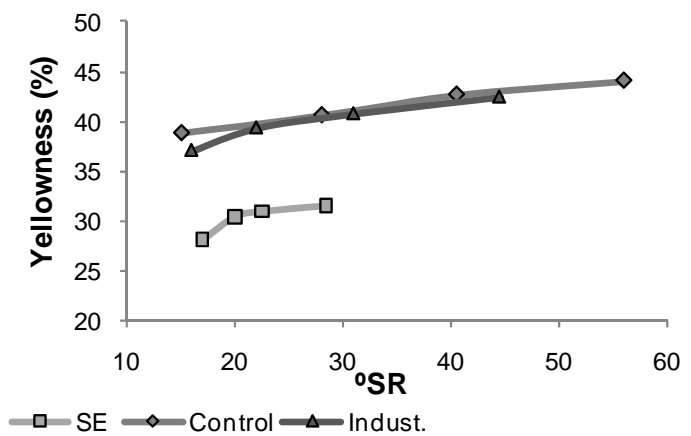

Fig. 6. Mechanical and optical properties of handsheets from exploded (SE- $\square$-), control (Control $\checkmark-$ ) and industrial (Indust. $\_$) pulps: a) apparent bulk density, b) tensile index, c) tear index, d) burst index, e) brightness, and f) yellowness index.

Based on the last results, it can be concluded that although exploded pulp showed slightly lower mechanical properties, optical properties were better than those of control and industrial pulps. Therefore, depending on the final paper use, exploded kraft pulp could be a good alternative to conventional kraft pulp.

\section{Accelerated Ageing}

The exploded, control, and industrial pulps were subjected to accelerated ageing in order to study the evolution of their optical and mechanical properties. Moreover, the effect of the steam explosion pretreatment on this evolution was evaluated. 
Table 5 shows the changes occurring in mechanical and optical properties with ageing. The reduction percentage for each mechanical or optical property was calculated as an average of the reductions (positive values in Table 5) or increases (negative values in Table 5) observed at different ${ }^{\circ} \mathrm{SR}$ in each pulp before and after the accelerated ageing.

Table 5. Reduction of Mechanical and Optical Properties in Exploded, Control and Industrial Pulps after Accelerated Ageing

\begin{tabular}{cccc}
\hline & SE8 & Control $\mathrm{A}+\mathrm{O}_{2}$ & Industrial \\
\hline Tensile Index & $-1.2 \%$ & $4.2 \%$ & $-3.8 \%$ \\
Tear Index & $8.3 \%$ & $10.7 \%$ & $16.2 \%$ \\
Burst Index & $-1.0 \%$ & $8.4 \%$ & $8.0 \%$ \\
Brightness & $4.6 \%$ & $9.2 \%$ & $2.7 \%$ \\
Yellowness Index & $-16.7 \%$ & $-2.0 \%$ & $-9.3 \%$ \\
\hline
\end{tabular}

After six days of accelerated ageing, the tensile index did not decrease significantly, while the tear and burst indexes were more affected. The steam exploded pulp showed the lowest decrease in brightness with ageing of all samples, but in the control pulp, the decrease was more pronounced than in the other pulps $(9.2 \%)$. However, the steam exploded pulp presented the biggest increase in yellowness index $(-16.7 \%)$, but even after ageing, the exploded pulp exhibited lower values of yellowness index than the control and industrial pulps.

From all these results, the steam explosion pulps exhibited a more favorable evolution with time, experiencing a lower decrease in their mechanical and optical properties after the accelerated ageing.

\section{CONCLUSIONS}

1. During steam explosion pretreatment most of the hemicelluloses could be recovered before pulping and converted in value-added products. In this way, a Eucalyptus globlus kraft pulp mill could be transformed into a forest biorefinery.

2. The steam explosion pretreatment reduced the cooking time from 50 to 20 minutes, obtaining a similar pulp and increasing the production rate of the mill.

3. Although the exploded pulp required more energy during refining, the mechanical properties were found to be just slightly lower and the optical properties higher than those from control and industrial pulps.

4. After an accelerated ageing both mechanical and optical properties were found to be more stable for exploded pulp than for control and industrial pulps.

\section{ACKNOWLEDGMENTS}

The authors wish to thank the Spanish Ministry of Science and Innovation for funding this study via Project CTQ 2006-00385, CTQ 2009-11268, the Ramón y Cajal Programme, and the FPI fellowship (BES2007 - 14528). 


\section{REFERENCES CITED}

Ahvazi, B., Radiotis, T., Bouchard, J., and Goel, K. (2007). "Chemical pulping of steamexploded mixed hardwood chips," J. Wood Chem. Technol. 27(2), 49-63.

Avellar, B. K., and Glasser, W. G. (1998). "Steam-assisted biomass fractionation. I. Process considerations and economic evaluation," Biomass Bioenerg. 14 (3), 205218.

Babcock, L.W. (1932). "Method of producing fermentable sugars and alcohol from wood," U.S. Patent No. 1855464.

Britt, K. W. (1970). Handbook of Pulp and Paper Technology, 2nd edn. Van Nostrand, New York.

Chua, M. G. S., and Wayman, M. (1979). "Characterization of autohydrolysis aspen (P.tremuloides) lignin. Part 1. Composition and molecular weight distribution of extracted autohydrolysis lignin," Can. J. Chem. 57, 1141-1149.

Delong, E. A. (1987). "Method of rendering lignin separable from cellulose and hemicelluloses and the product so produced," Canadian Pat. 1217765.

Garrote, G., Dominguez, H., and Parajo, J. C. (1999). "Mild autohydrolysis: An environmentally friendly technology for xylooligosaccharide production from wood," J. Chem. Technol. Biotechnol. 74, 1101-1109.

Gellersted, G., and Li, J. (1996). "An HPLC method for the quantitative determination of hexeneuronic acid groups in chemical pulps," Carbohydr. Res. 294, 41-51.

Heitner, C., Argyropoulos, D. S., Miles, K.D., Karnis, A., and Kerr, R. D. (1993). "Alkaline sulphite ultra-yield pulping of Aspen chips - A comparison of steamexplosion and conventional chemimechanical pulping," J. Pulp \& Paper Sci. 19(2), 58-70.

Jakobsons, J., Hortling, B., Erins, P., and Sundquist, J. (1995). "Characterization of alkali soluble fraction of steam exploded birch wood," Holzforschung 49, 51-59.

Josefsson, T., Lennholm, H., and Gellerstedt, G. (2001). "Changes in cellulose supramolecular structure and molecular weight distribution during steam explosion of aspen wood," Cellulose 8, 289-296.

Josefsson, T., Lennholm, H., and Gellerstedt, G. (2002). "Steam explosion of aspen wood. Characterization of reaction products," Holzforschung 53(3), 289-297.

Li, H., Saeed, A., Jahan, M.S., Ni, Y., and Van Heiningen, A. (2010). "Hemicellulose removal from hardwood chips in the pre-hydrolysis step of the kraft-based dissolving pulp production process," J. Wood Chem. Technol. 30, 48-60.

Manson, W. H. (1928). "Process and apparatus for disintegration of wood and the like," U.S. Patent No. 1655618.

Puls, J., Poutanen, K., Körner, H.U., and Viikari, L. (1985). "Biotechnical utilization of wood carbohydrates after steaming pretreatment," Appl. Microbiol. Biotechnol. 22, 416-423.

Overend, R. P., and Chornet, E. (1987). "Fractionation of lignocellulosics by steamaqueous pretreatments," Philos. Trans. R. Soc. London. A321, 523-536.

Ragauskas, A. J., Nagy, M., Kim, D. H., Eckert, C.A., Hallett, J. P., and Liotta, C. L. (2006). "From wood to fuels: integrating biofuels and pulp production," Indus.

Biotech. 2(1), 55-65. 
Ragnar, M. (2001). "On the importance of the structural composition of pulp for the selectivity of ozone and chlorine dioxide bleaching," Nordic Pulp Pap. Res. J. 16, 72.

Robert, D., Bardet, M., Lapierre, D., and Gellerstedt, G. (1988). "Structural changes in aspen lignin during steam explosion treatment," Cell. Chem. Technol. 22, 221-230.

San Martin, R., Perez, D., and Briones, R. (1995). "Simultaneous production of etanol and kraft pulp from pine (Pinus radiata) using steam explosion," Bioresource Technol. 53, 217-223.

Van Heiningen, A. (2006). "Converting a kraft pulp mill into an integrated biorefinery," Pulp Pap. Can. 107(6), 141-146.

Vuorinen, T., Fagerström, P., Buchert, J., Tenkanen M., and Teleman, A. (1999).

"Selective hydrolysis of hexenuronic acid groups and its application in ECF and TCF bleaching of kraft pulps," Pulp Pap. Sci. 25(5), 155-162.

Wafa Al-Dajani, W., and Tschirner, U.W. (2008). "Pre-extraction of hemicelluloses and subsequent kraft pulping. Part I: alkali extraction," Tappi J. 7(6), 3-8.

Wafa Al-Dajani, W., Tschirner, U.W., and Jense, T. (2009). "Pre-extraction of hemicelluloses and subsequent kraft pulping. Part II: Acid- and autochydrolysis," Tappi J. 30-37.

Wise, L. E., Murphy, M., and Daddieco, A. A. (1946). “Chlorite holocellulose, its fractionation and bearing on summative wood analysis and on studies on the hemicelluloses," Tech. Assoc. Pap. 29, 210-218.

Article submitted: November 11, 2010; Peer review completed: December 18, 2010; Revised version received and accepted: December 23, 2010; Published: December 28, 2010. 